A N N A L E S Annales de Bretagne et des Pays de l'Ouest

\title{
Dictionnaire des gens de couleur dans la France moderne
}

\author{
Dominique Le Page
}

\section{(2) OpenEdition}

Journals

Édition électronique

URL : http://journals.openedition.org/abpo/2798

DOI : $10.4000 /$ abpo. 2798

ISBN : 978-2-7535-3513-8

ISSN : 2108-6443

Éditeur

Presses universitaires de Rennes

Édition imprimée

Date de publication : 30 juin 2014

Pagination : 174-176

ISBN : 978-2-7535-3511-4

ISSN : 0399-0826

Référence électronique

Dominique Le Page, "Dictionnaire des gens de couleur dans la France moderne », Annales de Bretagne et des Pays de l'Ouest [En ligne], 121-2 | 2014, mis en ligne le 30 juin 2014, consulté le 10 décembre 2020. URL : http://journals.openedition.org/abpo/2798 ; DOI : https://doi.org/10.4000/abpo.2798 
d'un parcours à travers la constitution d'un vaste réseau de précieux informateurs et la difficulté à le faire fonctionner dans un univers politique où la faveur est essentielle, mais fragile, tant à la cour de France qu'au Sacré Collège. Cette dimension réticulaire dépasse évidemment le cas, emblématique, de Jean Du Bellay.

Philippe HAMON

NoËL, Erick (dir.), Dictionnaire des gens de couleur dans la France moderne, vol. II La Bretagne, Entrée par année, début XVI siècle-1792, Genève, Droz, 2013, 964 p.

Après un premier volume consacré à Paris et à son bassin, Erick Noël, professeur d'Histoire moderne à l'université des Antilles-Guyane, à qui l'on doit l'ouvrage Etre Noir en France au XVIII siècle (Paris, 2006) publie aux éditions Droz un second volume d'un Dictionnaire des gens de couleur consacré à la Bretagne grâce à la collaboration d'une dizaine de chercheurs - étudiants, enseignants, généalogistes venant principalement des universités de Nantes (Bernard Michon, Guy Saupin), des Antilles-Guyane (Dominique Rogers), d'Angers (Philippe Haudrère), du Maine (Sylvie Granger), de Washington (Sue Peabody), de Chicago (Robin Mitchell). L'ambition est d'établir une notice sur tous les individus - nègres, créoles, mulâtres, métis, quarterons... - qui ont séjourné en Bretagne des années 1550 jusqu'au début du XIX ${ }^{\mathrm{e}}$ siècle. À cette fin, des sources diverses et nombreuses ont été mobilisées aux Archives nationales et dans les principaux dépôts d'archives, départementaux et municipaux, de l'ouest : registres d'armement et de désarmement des navires, registres de baptêmes-sépultures des paroisses portuaires, archives notariales, Affiches générales de Bretagne de la chambre de commerce et d'industrie (années 1774-1785, cotes 364382). Des travaux précédents sont également mis à contribution, notamment celui d'Annick Le Douget, Juges, esclaves et négriers en Basse-Bretagne, 1750-1850 (2000) ou l'étude réalisée sous la direction d'Alain Croix sur Nantes (Nantais venus d'ailleurs, 2007). Les notices sont classées chronologiquement et par localité. L'ouvrage est complété par deux précieux index des gens de couleur et des maîtres. Ce dernier aurait pu être plus précis, en indiquant les fonctions des individus, ce qui aurait permis de faire un tri entre ceux qui portaient les mêmes noms et de faire apparaître les grandes catégories concernées par la présence noire en Bretagne.

Avec 7839 gens de couleur identifiés (sur plus de 15000 pour l'ensemble du royaume), la Bretagne apparaît comme une plaque tournante de la circulation des gens de couleur pendant l'Ancien Régime. Si leur présence est demeurée limitée pendant le $\mathrm{XvI}^{\mathrm{e}}$ siècle - le premier Noir repéré à Nantes est un "Maure d'Éthiopie ", Antonio, baptisé à Saint-Nicolas le 15 août 1553 -, elle s'est accrue dès les années 1640-1650 lors de l'engagement de Nantes dans le commerce négrier et s'est affirmée au cours d'un long xvIII ${ }^{\text {e }}$ siècle débutant dans les années 1690 et se poursuivant jusqu'à la période napoléonienne. Cet essor a été facilité par l'édit de 1716, inspiré par le futur maire de Nantes, Gérard Mellier, qui a accordé le droit d'amener des " esclaves nègres " en métropole pour servir comme domestiques ou apprendre un métier à la condition de les déclarer et de les renvoyer au bout d'un an (terme porté à trois ans en 1738). Le parlement de Rennes a entériné sans difficulté cette mesure à la différence de celui de Paris qui est demeuré attaché " au privilège affranchissant de la terre de France ". Si au fil du siècle, les autorités royales, par peur du métissage, ont cherché à limiter les arrivées des gens de couleur, les ordonnances prises en ce sens, comme celle de 1777 qui interdisait la venue de nouveaux noirs en France, ont été peu ou mal appliquées en raison de la résistance des négociants et ce n'est 
qu'avec la période révolutionnaire que l'on constate une véritable rupture dans les mouvements de population.

Parmi les villes d'accueil, la part de celle de Nantes est, sans surprise, prédominante avec 5901 cas identifiés entre la décennie 1650 et 1792. D'une cinquantaine de cas avant le traité d'Utrecht (1713), on en repère 1376 nouveaux pendant les années 1713-1742 et 4473 au cours du demi-siècle suivant, les guerres n'ayant donc pas perturbé les arrivées. Derrière Nantes, Lorient joue un rôle croissant avec 1464 individus loin devant Saint-Malo avec 224 cas. La part du reste de la Bretagne est faible avec 81 cas à Brest, 40 à Port-Louis et seulement 8 à Rennes. Ce sont surtout les milieux maritimes - capitaines, armateurs, négociants... - qui ont accueilli les gens de couleur alors que le monde des offices s'est montré plus réservé. Si l'on prend l'exemple de la chambre des comptes de Nantes, on ne trouve ainsi, après un rapide sondage, que quelques rares mentions de magistrats. Certains interviennent comme témoins lors d'un acte concernant un Noir : c'est le cas du maître des comptes Jacques Danguy (1723-1748) qui porte sur les fonts baptismaux en 1726, Hippolyte, nègre de Guinée ou du premier président des comptes Guillaume JeanBaptiste Becdelièvre (1716-1733) dans l'hôtel duquel - paroisse Saint-Vincent - est passé l'acte notarié par lequel le sieur Jacques Couillaud s'engage à envoyer le mulâtre François Lacroix, de la Martinique, à l'école pendant six mois. Ces présences relèvent de pratiques de charité comme le secours aux pauvres ou l'aide à la mise en apprentissage de jeunes adolescents. Seuls deux magistrats auraient possédé des esclaves noirs : François (ou René?) Bouchaud, sieur des Hérettes, auditeur des comptes (1731-1744), qui est propriétaire de François Jan, Maure, qu'il porte sur les fonts baptismaux en 1734 et Pierre Bellabre du Tellement, écuyer, domicilié à la Fosse, qui est dit propriétaire de deux esclaves domestiques alors qu'il n'est encore que receveur des fouages dans l'évêché de Nantes (il est ensuite maître des comptes - 1745-1748 - puis président - 1748-1752). L'emploi de gens de couleur semblait une tradition chez les Bellabre puisque son frère Mathurin, sénéchal et président du présidial de Nantes, était le maître en 1744 de L'Eveillé dit Favory qu'il a émancipé par testament et que leur père était propriétaire de l'esclave domestique Baptiste Loueda en 1733.

Dans les deux exemples, il s'agit de familles liées au monde maritime : le père de Bouchaud était armateur et consul des marchands à Nantes et son propre fils, François-Joseph, est devenu capitaine de vaisseau alors que l'oncle de Pierre Bellabre était capitaine en second du "Phénix ». C'est d'ailleurs lui qui a amené en métropole l'esclave Baptiste Loueda. Seules les familles de magistrats en lien avec le monde maritime et les colonies ont donc tenu à posséder des esclaves de couleur (cela est corroboré par l'exemple du général des finances Gatien Breton des Chapelles qui avait fait une partie de sa carrière à Saint-Domingue). Ce ne sont donc pas les élites dans leur ensemble qui ont été concernées par ce phénomène mais une partie d'entre elles, ce qui confirmerait la " frontière invisible " qui séparait le monde du négoce et de la robe dans la grande ville ligérienne. Ce constat est confirmé par le fait qu'à Rennes, ville parlementaire, la présence noire est très modeste.

Les destins de la majeure partie des gens de couleur identifiés demeurent inconnus d'autant que la plupart d'entre eux n'ont fait qu'un bref séjour sur les bords de la Loire, avant de repartir soit pour une autre ville - Nantes apparaît comme un lieu de transit notamment vers Paris - soit à bord d'un navire où ils pouvaient servir à l'occasion de membres d'équipage ou d'intermédiaires lors des opérations du trafic négrier. Certains sont demeurés plus longtemps, le temps de recevoir une instruction catholique et d'apprendre un métier - perruquier, cuisinier pour les hommes, couturière, lingère pour les femmes -. Seuls quelques-uns, qui font l'objet de notices plus fournies, ont laissé davantage de traces à l'image de Pierre la Violette qui aurait 
été le premier noir à faire souche à Nantes au début du XvIII ${ }^{\mathrm{e}}$ siècle grâce à la protection des puissantes familles Descazeaux et Darquistade ou d'Adélaïde Zaïre qui a osé en 1780, à Guérande, intenter un procès - qu'elle finit par remporter - aux parents de son ancien maître qui l'avait affranchie afin de récupérer ses effets. La ville de Nantes aurait vu la formation dans le quartier portuaire d'une petite communauté réunissant des esclaves affranchis exerçant une activité autonome et semble-t-il bien intégrée, ce qui démentirait l'idée de heurts avec la population locale.

Grâce à l'abondante information réunie et patiemment collectée, ce Dictionnaire qui sera sans nul doute enrichi dans les prochaines années - ce qui inviterait à constituer une base de données informatique -, donne une visibilité à une population condamnée jusque-là à l'ignorance ou au fantasme et constitue d'ores et déjà une solide base de travail pour tous ceux qui s'intéressent aux minorités dans le royaume de France sous l'Ancien Régime.

Dominique Le PAGE

SAVARY, Jacques, Le parfait négociant, RICHARD, Édouard (éd.), Genève, Droz, 2011, 2 vol., 2422 p.

Les ouvrages traitant des affaires de commerce constituent un genre littéraire dont l'apogée se situe aux XVII ${ }^{\mathrm{e}}$ et XVIII ${ }^{\mathrm{e}}$ siècle. Dans un souci d'universalité, propre à la période, les auteurs ont l'ambition de décrire le fonctionnement des échanges de manière à la fois exhaustive et synthétique. Le parfait négociant est l'œuvre de Jacques Savary (1622-1690), marchand-mercier parisien d'origine angevine qui, en 1656, après avoir fait fortune, abandonne son établissement pour acquérir une charge dans les domaines du roi grâce à l'appui de Fouquet. Après la disgrâce de son protecteur, il s'éloigne des affaires publiques mais il est rappelé par Colbert en 1670 et devient membre du conseil des réformes pour le commerce qui prépare l'ordonnance de 1673. Les mémoires qu'il a rédigées lors de ce travail le conduisent à publier Le parfait négociant dont la première édition date de 1675. L'ouvrage est un succès, assuré par de nombreuses rééditions (la dernière datant de 1800) et des traductions en anglais, néerlandais, allemand et italien lui donnent une grande notoriété.

L'œuvre de Savary est aujourd'hui rééditée par les éditions Droz de Genève en deux volumes. L'écriture de l'ouvrage a été corrigée et le texte modernisé par Édouard Richard avec la collaboration de Thierry Hamon, tous les deux enseignants en Histoire du droit à l'Université de Rennes 1. Un commentaire introductif suivi d'une bibliographie de 177 pages, est déjà en lui-même un ouvrage à part entière sur le travail de Savary. Édouard Richard y présente d'une plume alerte « quelques éléments pour cerner Le parfait négociant ". La lecture de ces commentaires sur le poids des mentalités, les vertus et les qualités du négociant, le fonctionnement des sociétés, l'utilisation de la lettre de change ou les affaires de faillites est passionnante. Les pages sur la lettre de change, sujet qui peut rebuter la plupart des lecteurs, sont d'une grande limpidité et sont à recommander à toute personne désireuse de comprendre les mécanismes des règlements commerciaux à l'époque moderne. Thierry Hamon complète cette introduction par un chapitre traitant de la dimension corporative chez Savary en illustrant son propos par de nombreux exemples bretons. Là aussi, les explications sur les cadres juridiques, l'apprentissage où les instructions pour accéder à la maîtrise sont remarquables de par leur clarté et leur intérêt. 